PROCEEDINGS OF THE

AMERICAN MATHEMATICAL SOCIETY

Volume 136, Number 10, October 2008, Pages 3529-3537

S 0002-9939(08)09562-2

Article electronically published on May 29, 2008

\title{
ALTERNATE SIGNS BANACH-SAKS PROPERTY AND REAL INTERPOLATION OF OPERATORS
}

\author{
ANDRZEJ KRYCZKA
}

(Communicated by N. Tomczak-Jaegermann)

\begin{abstract}
In the space of bounded linear operators acting between Banach spaces we define a seminorm vanishing on the subspace of operators having the alternate signs Banach-Saks property. We obtain logarithmically convex-type estimates of the seminorm for operators interpolated by the Lions-Peetre real method. In particular, the estimates show that the alternate signs BanachSaks property is inherited from a space of an interpolation pair $\left(A_{0}, A_{1}\right)$ to the real interpolation spaces $A_{\theta, p}$ with respect to $\left(A_{0}, A_{1}\right)$ for all $0<\theta<1$ and $1<p<\infty$.
\end{abstract}

\section{INTRODUCTION}

A bounded linear operator $T: X \rightarrow Y$ acting between Banach spaces is said to have the Banach-Saks (BS) property if every bounded sequence $\left(x_{n}\right)$ in $X$ contains a subsequence $\left(x_{n}^{\prime}\right)$ such that the Cesàro means of $\left(T x_{n}^{\prime}\right)$ converge in $Y$. If we restrict this definition to all weakly null sequences $\left(x_{n}\right)$ in $X$, we say that $T$ has the weak Banach-Saks (WBS) property or the Banach-Saks-Rosenthal property. We say that $T$ has the alternate signs Banach-Saks (ABS) property if every bounded sequence $\left(x_{n}\right)$ in $X$ contains a subsequence $\left(x_{n}^{\prime}\right)$ such that the Cesàro means of $\left((-1)^{n} T x_{n}^{\prime}\right)$ converge in $Y$.

A Banach space $X$ is said to have the BS, WBS or ABS property if the corresponding property is possessed by the identity operator $I: X \rightarrow X$. The ABS property is weaker than the BS property and stronger than the WBS property. The relations are strict: $c_{0}$ has the ABS property but does not have the BS property; $l_{1}$ has the WBS property but does not have the ABS property. For a detailed study of these properties we refer the reader to [2].

A natural question is the behavior of Banach-Saks properties under interpolation. Beauzamy 2 proved that if $\left(A_{0}, A_{1}\right)$ is an interpolation pair such that $A_{0}$ is continuously embedded in $A_{1}$ and the embedding has the ABS property, then the real interpolation spaces $A_{\theta, p}$ with respect to $\left(A_{0}, A_{1}\right)$ have the ABS property for all $0<\theta<1$ and $1<p<\infty$. This in turn served to show that every operator with the $\mathrm{BS}$ or $\mathrm{ABS}$ property factors through a space with the same property (see also 4]). Heinrich [9] proved that if the embedding $I: A_{0} \cap A_{1} \rightarrow A_{0}+A_{1}$ has the BS

Received by the editors July 11, 2007.

2000 Mathematics Subject Classification. Primary 46B70, 47A30; Secondary 47B10.

Key words and phrases. Alternate signs Banach-Saks property, real interpolation method, spreading model.

(C)2008 American Mathematical Society Reverts to public domain 28 years from publication 
property, then so has $A_{\theta, p}$ with respect to $\left(A_{0}, A_{1}\right)$ for all $0<\theta<1$ and $1<p<\infty$ (see also [3]).

Another line of investigation has a quantitative character. In this approach, we focus on inequalities which may also provide qualitative information. Astala and Tylli introduced the so-called outer [1] and inner [14] measures for bounded linear operators. These measures are related to certain operator ideals such as compact, weakly compact, Rosenthal operators and operators with the BS property. Roughly speaking, they measure the deviation of an operator from a given ideal. The interpolation properties of these uniform measures have been investigated in detail by Cobos, Manzano and Martínez in [6] and [7.

The aim of this paper is to find a measure of deviation from the ABS property with good interpolation properties. Our work is motivated by [10, where similar results for a measure of weak noncompactness were obtained. Using Beauzamy's geometric characterization of the ABS property, we define in the space of bounded linear operators a seminorm vanishing on the subspace of operators having the ABS property. As in Beauzamy's works, the key tool here is the spreading model of Brunel and Sucheston. We obtain logarithmically convex (up to a constant) estimates of the seminorm for operators interpolated by the real method. In particular, the inequalities show that the ABS property is hereditary under real interpolation. It is worth pointing out that the types of estimates obtained in this paper are significantly different from that of [6] and [7].

The cardinality of a subset $A \subset \mathbb{N}$ will be denoted by $|A|$. We write $\mathrm{B}(X)$ for the open unit ball of a Banach space $X$. By $\mathcal{L}(X, Y)$ we denote the space of all bounded linear operators acting between Banach spaces $X$ and $Y$. The subspace of $\mathcal{L}(X, Y)$ consisting of all operators having the ABS property will be denoted by $\mathcal{A B S}(X, Y)$.

\section{ABS PROPERTY AND SPREADING MODELS}

One of the basic results on Banach-Saks properties is the following one of Rosenthal [13]: if a Banach space $X$ does not have the WBS property, then there exist a number $\delta>0$ and a bounded sequence $\left(x_{n}\right)$ in $X$ such that for all $k \in \mathbb{N}$, all subsets $A \subset \mathbb{N}$ with $|A|=2^{k}$ and $k \leq \min A$, and all sequences of scalars $\left(c_{n}\right)$, we have $\left\|\sum_{n \in A} c_{n} x_{n}\right\| \geq \delta \sum_{n \in A}\left|c_{n}\right|$.

Beauzamy 2 proved that the above implication turns into an equivalence if we replace the WBS property by the ABS property. The starting point of our considerations is another of Beauzamy's results: a Banach space $X$ does not have the ABS property if and only if there exist a number $\delta>0$ and a bounded sequence $\left(x_{n}\right)$ in $X$ such that for all finite subsets $A \subset \mathbb{N}$ and all sequences of signs $\left(\epsilon_{n}\right)$, $\epsilon_{n}= \pm 1$ for all $n$, we have $\left\|\sum_{n \in A} \epsilon_{n} x_{n}\right\| \geq \delta|A|$.

We introduce the following relations: $\left(y_{n}\right)$ is a sequence of successive variable signs means ( $\mathrm{svsm}$ ) for $\left(x_{n}\right)$ if there exist $m \in \mathbb{N}$, a sequence $\left(A_{n}\right)$ of finite subsets of $\mathbb{N}$ with $\max A_{n}<\min A_{n+1}$ and $\left|A_{n}\right|=m$ for all $n$, and a sequence of signs $\left(\epsilon_{n}\right)$, such that $y_{n}=m^{-1} \sum_{k \in A_{n}} \epsilon_{k} x_{k}$ for all $n$; if in this definition $\epsilon_{n}=1$ for all $n$, then $\left(y_{n}\right)$ is called a sequence of successive arithmetic means (sam) for $\left(x_{n}\right)$. Since all sets $A_{n}$ are equipollent, the relation svsm is transitive. 
Definition 2.1. Let $\left(x_{n}\right)$ be a bounded sequence in a Banach space $X$. Define

$$
\phi_{v s m}\left(x_{n}\right)=\inf \left\||A|^{-1} \sum_{n \in A} \epsilon_{n} x_{n}\right\|, \quad \phi_{a m}\left(x_{n}\right)=\inf \left\||A|^{-1} \sum_{n \in A} x_{n}\right\|,
$$

the infimum for $\phi_{v s m}$ being taken over all finite subsets $A \subset \mathbb{N}$ and all sequences of signs $\left(\epsilon_{n}\right)$, the infimum for $\phi_{\text {am }}$ being taken over all finite subsets $A \subset \mathbb{N}$.

If $\left(y_{n}\right)$ is a sequence of svsm for $\left(x_{n}\right)$, in particular, if $\left(y_{n}\right)$ is a subsequence of $\left(x_{n}\right)$ or $\left(y_{n}\right)$ is a sequence of sam for $\left(x_{n}\right)$, then $\phi_{v s m}\left(x_{n}\right) \leq \phi_{v s m}\left(y_{n}\right)$.

We recall the result of Brunel and Sucheston [5] on extraction of 'good' subsequences, following [2. We apply this result to construct 'good' sequences of svsm and sam for a given bounded sequence.

Proposition 2.2. Let $\left(x_{n}\right)$ be a bounded sequence in a Banach space $X$. There exist a subsequence $\left(x_{n}^{\prime}\right)$ of $\left(x_{n}\right)$ and a seminorm $L$ in the set $S$ of all finite sequences of scalars (real or complex), with the following property: for every $\varepsilon>0$ and every $a=\left(a_{1}, \ldots, a_{m}\right) \in S$ there exists $v \in \mathbb{N}$ such that, if $v \leq n_{1}<\ldots<n_{m}$, then $\left|\left\|\sum_{i=1}^{m} a_{i} x_{n_{i}}^{\prime}\right\|-L(a)\right|<\varepsilon$.

If $\left(x_{n}\right)$ has no Cauchy subsequence, the formula $\left\|a_{1} x_{1}^{\prime}+\cdots+a_{m} x_{m}^{\prime}\right\|_{E}=L(a)$, $a=\left(a_{1}, \ldots, a_{m}\right)$, defines a norm in the space spanned by vectors $x_{n}^{\prime}$. Let $E$ be the completion of $\operatorname{span}\left\{x_{n}^{\prime}\right\}$ under this norm. The space $E$ is called the spreading model of $X$ built on $\left(x_{n}\right)$. The sequence $\left(x_{n}^{\prime}\right)$ is called the fundamental sequence of $E$. The norm of $E$ is invariant under spreading; that is, $\left\|a_{1} x_{1}^{\prime}+\cdots+a_{m} x_{m}^{\prime}\right\|_{E}=$ $\left\|a_{1} x_{n_{1}}^{\prime}+\cdots+a_{m} x_{n_{m}}^{\prime}\right\|_{E}$ for all $n_{1}<\ldots<n_{m}$.

The next proposition will play a key role in our considerations. Its assertion is related to property $\left(P_{1}^{\prime}\right)$ of $[2$. In the proof, we follow the main line of the proof of Theorem II.2 of [2].

Proposition 2.3. Let $\left(x_{n}\right)$ be a bounded sequence in a Banach space $X$. Then for every $\varepsilon>0$ there exist a sequence $\left(y_{n}\right)$ of svsm for $\left(x_{n}\right)$ and a sequence $\left(v_{n}\right)$ of sam for $\left(x_{n}\right)$ such that for all finite subsets $A \subset \mathbb{N}$ and all sequences of signs $\left(\epsilon_{n}\right)$,

$$
\left\||A|^{-1} \sum_{n \in A} \epsilon_{n} y_{n}\right\| \leq \phi_{v s m}\left(y_{n}\right)+\varepsilon, \quad\left\||A|^{-1} \sum_{n \in A} v_{n}\right\| \leq \phi_{a m}\left(v_{n}\right)+\varepsilon .
$$

Proof. We prove the assertion for the relation svsm. The proof for the relation sam is almost the same. Fix $\varepsilon>0$. First assume that $\left(x_{n}\right)$ contains a Cauchy subsequence $\left(x_{n}^{\prime}\right)$. Put $y_{n}=\left(x_{2 n}^{\prime}-x_{2 n-1}^{\prime}\right) / 2$. Ignoring a finite number of terms of $\left(y_{n}\right)$, we see that $\left(y_{n}\right)$ satisfies the assertion.

Now assume that $\left(x_{n}\right)$ has no Cauchy subsequence. Let a subsequence $\left(x_{n}^{\prime}\right)$ of $\left(x_{n}\right)$ be the fundamental sequence of the spreading model $E$ built on $\left(x_{n}\right)$, given by Proposition 2.2, Taking $\left(x_{n}^{\prime}\right)$ in the norm $\|\cdot\|_{E}$, we put $K=\phi_{v s m}\left(x_{n}^{\prime}\right)$. There exists $z=m^{-1} \sum_{i=1}^{m} \epsilon_{i}^{\prime} x_{n_{i}}^{\prime}$, where $n_{1}<\ldots<n_{m}$ and $\epsilon_{1}^{\prime}, \ldots, \epsilon_{m}^{\prime}$ is a finite sequence of signs, such that $K \leq\|z\|_{E} \leq K+\varepsilon / 4$. Put $z_{n}=m^{-1} \sum_{i=1}^{m} \epsilon_{i}^{\prime} x_{(n-1) m+i}^{\prime}$ for every $n \in \mathbb{N}$. Since $\|\cdot\|_{E}$ is invariant under spreading, $K \leq\left\|z_{n}\right\|_{E} \leq K+\varepsilon / 4$. Clearly, for all finite subsets $A \subset \mathbb{N}$ and all sequences of signs $\left(\epsilon_{n}\right)$,

$$
K \leq\left\||A|^{-1} \sum_{n \in A} \epsilon_{n} z_{n}\right\|_{E} \leq K+\varepsilon / 4 .
$$


Let $k \in \mathbb{N}$. Applying Proposition 2.2, we get $n_{k}$ such that if $B \subset \mathbb{N}$ with $|B| \leq 2^{k}$ and $n_{k} \leq \min B$, then for all sequences of signs $\left(\epsilon_{n}\right)$,

$$
\left\|\left.|| B\right|^{-1} \sum_{n \in B} \epsilon_{n} z_{n}\right\|-\left\||B|^{-1} \sum_{n \in B} \epsilon_{n} z_{n}\right\|_{E} \mid<\varepsilon / 4
$$

We may assume that $n_{k}<n_{k+1}$ for all $k$. It follows that for the sequence $\left(z_{k}^{\prime}\right)$ with $z_{k}^{\prime}=z_{n_{k}}$, all $B \subset \mathbb{N}$ with $|B| \leq 2^{k}$ and $k \leq \min B$, and all sequences of signs $\left(\epsilon_{n}\right)$,

$$
K-\varepsilon / 4 \leq\left\||B|^{-1} \sum_{n \in B} \epsilon_{n} z_{n}^{\prime}\right\| \leq K+\varepsilon / 2 .
$$

Let $A \subset \mathbb{N}$ be finite and $A_{0}=\left\{n \in A: n<\log _{2}|A|\right\}$. Then

$$
\left\|\sum_{n \in A_{0}} \epsilon_{n} z_{n}^{\prime}\right\| \leq\left|A_{0}\right|(K+\varepsilon / 2) \quad \text { and } \quad\left\|\sum_{n \in A \backslash A_{0}} \epsilon_{n} z_{n}^{\prime}\right\| \geq\left|A \backslash A_{0}\right|(K-\varepsilon / 4) .
$$

Of course, we assume that the sum over the empty set is 0 . Consequently,

$$
\begin{aligned}
\left\||A|^{-1} \sum_{n \in A} \epsilon_{n} z_{n}^{\prime}\right\| & \geq\left\||A|^{-1} \sum_{n \in A \backslash A_{0}} \epsilon_{n} z_{n}^{\prime}\right\|-\left\||A|^{-1} \sum_{n \in A_{0}} \epsilon_{n} z_{n}^{\prime}\right\| \\
& \geq K-\varepsilon / 4-\left|A_{0}\right||A|^{-1}(2 K+\varepsilon / 4) .
\end{aligned}
$$

There is an $m_{0} \in \mathbb{N}$ such that if $|A| \geq m_{0}$, then $\left|A_{0}\right||A|^{-1}(2 K+\varepsilon / 4) \leq \varepsilon / 4$. Then

$$
K-\varepsilon / 2 \leq\left\||A|^{-1} \sum_{n \in A} \epsilon_{n} z_{n}^{\prime}\right\| \leq K+\varepsilon / 2 .
$$

Let $y_{n}=m_{0}^{-1} \sum_{i=1}^{m_{0}} z_{(n-1) m_{0}+i}^{\prime}$ for every $n \in \mathbb{N}$. Then for all finite subsets $A \subset \mathbb{N}$ and all sequences of signs $\left(\epsilon_{n}\right)$,

$$
K+\varepsilon / 2 \geq\left\||A|^{-1} \sum_{n \in A} \epsilon_{n} y_{n}\right\| \geq\left\||A|^{-1} m_{0}^{-1} \sum_{n \in A} \sum_{i=1}^{m_{0}} \epsilon_{n} z_{(n-1) m_{0}+i}^{\prime}\right\| \geq K-\varepsilon / 2 .
$$

Thus $\left\||A|^{-1} \sum_{n \in A} \epsilon_{n} y_{n}\right\| \leq \phi_{v s m}\left(y_{n}\right)+\varepsilon$ for all finite subsets $A \subset \mathbb{N}$ and all sequences of signs $\left(\epsilon_{n}\right)$. Of course, $\left(y_{n}\right)$ is a sequence of svsm for $\left(x_{n}\right)$.

Definition 2.4. Let $X, Y$ be Banach spaces and $T \in \mathcal{L}(X, Y)$. Define

$$
\Phi_{A B S}(T)=\sup \left\{\phi_{v s m}\left(T x_{n}\right):\left(x_{n}\right) \subset \mathrm{B}(X)\right\} .
$$

Proposition 2.5. $\Phi_{A B S}$ is a seminorm in $\mathcal{L}(X, Y) . \Phi_{A B S}(T)=0$ if and only if $T \in A \mathcal{B S}(X, Y)$.

Proof. Clearly, $\Phi_{A B S}(\lambda T)=|\lambda| \Phi_{A B S}(T)$ for all scalars $\lambda$. We show that for all $S, T \in \mathcal{L}(X, Y), \Phi_{A B S}(S+T) \leq \Phi_{A B S}(S)+\Phi_{A B S}(T)$. Let $\varepsilon>0$ and $\left(x_{n}\right) \subset \mathrm{B}(X)$. By Proposition 2.3, there exists a sequence $\left(x_{n}^{\prime}\right)$ of svsm for $\left(x_{n}\right)$ such that for the sequence $\left(S x_{n}^{\prime}\right)$ of svsm for $\left(S x_{n}\right)$,

$$
\left\||A|^{-1} \sum_{n \in A} \epsilon_{n} S x_{n}^{\prime}\right\| \leq \phi_{v s m}\left(S x_{n}^{\prime}\right)+\varepsilon
$$


for all finite subsets $A \subset \mathbb{N}$ and all sequences of signs $\left(\epsilon_{n}\right)$. Again applying Proposition 2.3, we get a sequence $\left(x_{n}^{\prime \prime}\right)$ of svsm for $\left(x_{n}^{\prime}\right)$, such that for all finite subsets $A \subset \mathbb{N}$ and all sequences of signs $\left(\epsilon_{n}\right)$,

$$
\left\||A|^{-1} \sum_{n \in A} \epsilon_{n} T x_{n}^{\prime \prime}\right\| \leq \phi_{v s m}\left(T x_{n}^{\prime \prime}\right)+\varepsilon .
$$

Since the relation svsm is transitive,

$$
\begin{aligned}
\phi_{v s m}\left((S+T) x_{n}\right) & \leq \phi_{v s m}\left((S+T) x_{n}^{\prime \prime}\right) \leq\left\||A|^{-1} \sum_{n \in A} \epsilon_{n}(S+T) x_{n}^{\prime \prime}\right\| \\
& \leq\left\||A|^{-1} \sum_{n \in A} \epsilon_{n} S x_{n}^{\prime \prime}\right\|+\left\||A|^{-1} \sum_{n \in A} \epsilon_{n} T x_{n}^{\prime \prime}\right\| \\
& \leq \phi_{v s m}\left(S x_{n}^{\prime}\right)+\phi_{v s m}\left(T x_{n}^{\prime \prime}\right)+2 \varepsilon \leq \Phi_{A B S}(S)+\Phi_{A B S}(T)+2 \varepsilon .
\end{aligned}
$$

By an arbitrary choice of $\varepsilon>0$ and $\left(x_{n}\right) \subset \mathrm{B}(X)$, we obtain the conclusion.

By Theorem IV.2 of [2], $T$ has the ABS property if and only if for every bounded sequence $\left(x_{n}\right)$ in $X$ there exist a subsequence $\left(x_{n}^{\prime}\right)$ of $\left(x_{n}\right)$ and a sequence of signs $\left(\epsilon_{n}\right)$ such that the Cesàro means of $\left(\epsilon_{n} T x_{n}^{\prime}\right)$ converge to 0 in $Y$. From this and by Theorem III.1 of [2], $T$ has the ABS property if and only if for every bounded sequence $\left(x_{n}\right)$ in $X, \phi_{v s m}\left(T x_{n}\right)=0$. By positive homogeneity of $\Phi_{A B S}, T$ has the ABS property if and only if $\Phi_{A B S}(T)=0$.

\section{ABS PROPERTY AND $l_{p}(X)$ SPACES}

Let $X$ be a Banach space, $1<p<\infty$ and let $\left(e_{i}\right)$ be the unit vector basis of $l_{p}$. We denote by $l_{p}(X)$ the Banach space of all sequences $x=(x(i))$ such that $x(i) \in X$ for every $i \in \mathbb{N}$ and

$$
\|x\|_{l_{p}(X)}=\left\|\sum_{i=1}^{\infty}\right\| x(i)\left\|_{X} e_{i}\right\|_{l_{p}}<\infty .
$$

In the sequel, we also deal with $l_{p}(X)$ of the families $(x(i))_{i \in \mathbb{Z}}$ indexed by integers.

Partington [12] proved that $l_{p}(X), 1<p<\infty$, has the BS property if and only if so has $X$ (in fact, a more general setting of direct sums was used). We use similar arguments as in the proof of Theorem 3 of [12] to show the next lemma.

Lemma 3.1. Let $X$ be a Banach space and $\left(x_{n}\right)$ a bounded sequence in $l_{p}(X)$, $1<p<\infty$. Then for every $\varepsilon>0$ there exist $m \in \mathbb{N}$ and a sequence $\left(y_{n}\right)$ of sam for $\left(x_{n}\right)$ such that for all finite subsets $A \subset \mathbb{N}$ and all sequences of signs $\left(\epsilon_{n}\right)$,

$$
\left\|\sum_{i=m+1}^{\infty}\right\||A|^{-1} \sum_{n \in A} \epsilon_{n} y_{n}(i)\left\|_{X} e_{i}\right\|_{l_{p}}<\varepsilon .
$$

Proof. For $x_{n}=\left(x_{n}(i)\right) \in l_{p}(X)$, let $t_{n}=\sum_{i=1}^{\infty}\left\|x_{n}(i)\right\|_{X} e_{i} \in l_{p}$. Since $l_{p}$ has the BS property, by Erdös-Magidor's theorem in [8], there exists a subsequence $\left(t_{n}^{\prime}\right)$ of $\left(t_{n}\right)$ such that the Cesàro means of all subsequences of $\left(t_{n}^{\prime}\right)$ converge to the same limit $t$ in $l_{p}$. Then $\phi_{a m}\left(s_{n}-t\right)=0$ for every sequence $\left(s_{n}\right)$ of sam for $\left(t_{n}^{\prime}\right)$. By 
Proposition 2.3. there exists a sequence $\left(s_{n}\right)$ of sam for $\left(t_{n}^{\prime}\right)$ such that for every finite subset $A \subset \mathbb{N}$,

$$
\left\||A|^{-1} \sum_{n \in A} s_{n}-t\right\|_{l_{p}}<\varepsilon / 2
$$

There exist $k_{0} \in \mathbb{N}$ and a sequence $\left(A_{n}\right)$ of finite subsets of $\mathbb{N}$ with $\max A_{n}<$ $\min A_{n+1}$ and $\left|A_{n}\right|=k_{0}$ for all $n$ such that $s_{n}=k_{0}^{-1} \sum_{k \in A_{n}} t_{k}^{\prime}$. Let $\left(y_{n}\right)$ be the corresponding sequence of sam for $\left(x_{n}\right)$. That is, first we take the subsequence $\left(x_{n}^{\prime}\right)$ of $\left(x_{n}\right)$ such that $t_{n}^{\prime}=\sum_{i=1}^{\infty}\left\|x_{n}^{\prime}(i)\right\|_{X} e_{i}$, and then we put $y_{n}=k_{0}^{-1} \sum_{k \in A_{n}} x_{k}^{\prime}$. Let $t=\sum_{i=1}^{\infty} \alpha_{i} e_{i}$ and let $m \in \mathbb{N}$ satisfy $\left\|\sum_{i=m+1}^{\infty} \alpha_{i} e_{i}\right\|_{l_{p}}<\varepsilon / 2$. Then for every finite subset $A \subset \mathbb{N}$,

$$
\left\|\sum_{i=m+1}^{\infty}\left(|A|^{-1} \sum_{n \in A} k_{0}^{-1} \sum_{k \in A_{n}}\left\|x_{k}^{\prime}(i)\right\|_{X}-\alpha_{i}\right) e_{i}\right\|_{l_{p}}<\varepsilon / 2 .
$$

It follows that

$$
\left\|\sum_{i=m+1}^{\infty}\left(|A|^{-1} \sum_{n \in A} k_{0}^{-1} \sum_{k \in A_{n}}\left\|x_{k}^{\prime}(i)\right\|_{X}\right) e_{i}\right\|_{l_{p}}<\varepsilon .
$$

By hyperorthogonality of the basis $\left(e_{i}\right)$, for all sequences of signs $\left(\epsilon_{n}\right)$,

$$
\left\|\sum_{i=m+1}^{\infty}\right\||A|^{-1} \sum_{n \in A} \epsilon_{n} y_{n}(i)\left\|_{X} e_{i}\right\|_{l_{p}}<\varepsilon .
$$

The next result is crucial for the interpolation properties of $\Phi_{A B S}$. This is a counterpart of Theorem 3.6 of [10] proved for a measure of weak noncompactness.

Theorem 3.2. Let $X, Y$ be Banach spaces and $1<p<\infty$. If $T \in \mathcal{L}(X, Y)$ and if $\widetilde{T} \in \mathcal{L}\left(l_{p}(X), l_{p}(Y)\right)$ is given by $\widetilde{T} x=(T x(i))$ for every $x=(x(i))$, then $\Phi_{A B S}(T)=\Phi_{A B S}(\widetilde{T})$.

Proof. Since $l_{p}(X)$ contains isometric copies of $X, \Phi_{A B S}(T) \leq \Phi_{A B S}(\widetilde{T})$. Fix $\varepsilon>0$. There exists $\left(x_{n}\right) \subset \mathrm{B}\left(l_{p}(X)\right)$ such that $\Phi_{A B S}(\widetilde{T})-\varepsilon \leq \phi_{v s m}\left(\widetilde{T} x_{n}\right)$. By Lemma 3.1 there exist $m \in \mathbb{N}$ and a sequence $\left(x_{n}^{\prime}\right)$ of sam for $\left(x_{n}\right)$ such that for the sequence $\left(\widetilde{T} x_{n}^{\prime}\right)$ of sam for $\left(\widetilde{T} x_{n}\right)$, and for all finite subsets $A \subset \mathbb{N}$ and all sequences of signs $\left(\epsilon_{n}\right)$,

$$
\left\|\sum_{i=m+1}^{\infty}\right\||A|^{-1} \sum_{n \in A} \epsilon_{n} T x_{n}^{\prime}(i)\left\|_{Y} e_{i}\right\|_{l_{p}}<\varepsilon .
$$

There exists a subsequence $\left(x_{n}^{\prime \prime}\right)$ of $\left(x_{n}^{\prime}\right)$ such that for each $1 \leq i \leq m$ the limit $\beta_{i}=\lim _{n}\left\|x_{n}^{\prime \prime}(i)\right\|_{X}$ exists and $\left\|x_{n}^{\prime \prime}(i)\right\|_{X}<\beta_{i}+\varepsilon / m$ for every $n$. Putting $v_{n}(i)=\left(\beta_{i}+\varepsilon / m\right)^{-1} T x_{n}^{\prime \prime}(i)$, we have $\left(v_{n}(i)\right) \subset T(\mathrm{~B}(X))$ for every $1 \leq i \leq m$.

By Proposition 2.3, there exists a sequence $\left(x_{n}^{1}\right)$ of svsm for $\left(x_{n}^{\prime \prime}\right)$ such that for the sequence $\left(v_{n}^{1}(1)\right)$ of svsm for $\left(v_{n}(1)\right)$, where $v_{n}^{1}(i)=\left(\beta_{i}+\varepsilon / m\right)^{-1} T x_{n}^{1}(i)$, $1 \leq i \leq m$, we have

$$
\left\||A|^{-1} \sum_{n \in A} \epsilon_{n} v_{n}^{1}(1)\right\|_{Y} \leq \phi_{v s m}\left(v_{n}^{1}(1)\right)+\varepsilon
$$

for all finite subsets $A \subset \mathbb{N}$ and all sequences of signs $\left(\epsilon_{n}\right)$. 
Proceeding in this way consecutively for $i=2, \ldots, m$, in the $k$ th step, we obtain a sequence $\left(x_{n}^{k}\right)$ of svsm for $\left(x_{n}^{k-1}\right)$ such that for the sequence $\left(v_{n}^{k}(k)\right)$ of svsm for $\left(v_{n}^{k-1}(k)\right)$, where $v_{n}^{k}(i)=\left(\beta_{i}+\varepsilon / m\right)^{-1} T x_{n}^{k}(i), 1 \leq i \leq m$, we have

$$
\left\||A|^{-1} \sum_{n \in A} \epsilon_{n} v_{n}^{k}(k)\right\|_{Y} \leq \phi_{v s m}\left(v_{n}^{k}(k)\right)+\varepsilon
$$

for all finite subsets $A \subset \mathbb{N}$ and all sequences of signs $\left(\epsilon_{n}\right)$. In this way, all sequences $\left(v_{n}^{m}(i)\right), 1 \leq i \leq m$, are built on the common sequence $\left(x_{n}^{m}\right)$ of svsm for $\left(x_{n}\right)$, and for all finite subsets $A \subset \mathbb{N}$ and all sequences of signs $\left(\epsilon_{n}\right)$,

$$
\left\||A|^{-1} \sum_{n \in A} \epsilon_{n} v_{n}^{m}(i)\right\|_{Y} \leq \phi_{v s m}\left(v_{n}^{m}(i)\right)+\varepsilon, \quad 1 \leq i \leq m .
$$

It follows that

$$
\begin{aligned}
\phi_{v s m}\left(\widetilde{T} x_{n}\right) & \leq \phi_{v s m}\left(\widetilde{T} x_{n}^{m}\right) \leq\left\|\sum_{i=1}^{m}\right\||A|^{-1} \sum_{n \in A} \epsilon_{n} T x_{n}^{m}(i)\left\|_{Y} e_{i}\right\|_{l_{p}}+\varepsilon \\
& =\left\|\sum_{i=1}^{m}\right\|\left(\beta_{i}+\varepsilon / m\right)|A|^{-1} \sum_{n \in A} \epsilon_{n} v_{n}^{m}(i)\left\|_{Y} e_{i}\right\|_{l_{p}}+\varepsilon \\
& \leq\left\|\sum_{i=1}^{m}\left|\beta_{i}+\varepsilon / m\right| e_{i}\right\|_{l_{p}} \max _{1 \leq i \leq m}\left\||A|^{-1} \sum_{n \in A} \epsilon_{n} v_{n}^{m}(i)\right\|_{Y}+\varepsilon \\
& \leq\left(1+\varepsilon m^{1 / p-1}\right)_{1 \leq i \leq m}\left\{\phi_{v s m}\left(v_{n}^{m}(i)\right)+\varepsilon\right\}+\varepsilon .
\end{aligned}
$$

There exists $1 \leq j \leq m$ such that $\phi_{v s m}\left(v_{n}^{m}(j)\right)=\max _{1 \leq i \leq m} \phi_{v s m}\left(v_{n}^{m}(i)\right)$. Since $\left(v_{n}^{m}(j)\right)$ is a sequence of svsm for $\left(v_{n}(j)\right)$, we have $\left(v_{n}^{m}(j)\right) \subset T(\mathrm{~B}(X))$ and consequently,

$$
\Phi_{A B S}(\widetilde{T})-2 \varepsilon \leq\left(1+\varepsilon m^{1 / p-1}\right)\left(\Phi_{A B S}(T)+\varepsilon\right)
$$

Letting $\varepsilon \rightarrow 0$, we get $\Phi_{A B S}(\widetilde{T}) \leq \Phi_{A B S}(T)$.

Corollary 3.3. The space $l_{p}(X), 1<p<\infty$, has the ABS property if and only if $X$ has the $A B S$ property.

\section{ABS PROPERTY AND REAL INTERPOLATION}

We recall briefly some basic definitions and facts concerning real interpolation. For a thorough treatment we refer to 11 .

If two Banach spaces $A_{0}$ and $A_{1}$ are linearly and continuously embedded in a common Hausdorff topological vector space $V$, we call $\vec{A}=\left(A_{0}, A_{1}\right)$ an interpolation pair. Then $\Delta(\vec{A})=A_{0} \cap A_{1}, \Sigma(\vec{A})=A_{0}+A_{1}$ are Banach spaces with norms

$$
\|a\|_{\Delta(\vec{A})}=\max \left\{\|a\|_{A_{0}},\|a\|_{A_{1}}\right\}, \quad\|a\|_{\Sigma(\vec{A})}=\inf \left\{\left\|a_{0}\right\|_{A_{0}}+\left\|a_{1}\right\|_{A_{1}}: a_{0}+a_{1}=a\right\} .
$$

We consider a discrete method of construction of the real interpolation spaces of Lions and Peetre [11]. For $0<\theta<1$ and $1<p<\infty$, let

$$
A_{\theta, p}=\left\{a \in \Sigma(\vec{A}):\|a\|_{A_{\theta, p}}<\infty\right\}
$$


where

$$
\|a\|_{A_{\theta, p}}=\inf \max \left\{\left\|\left(2^{i \theta} a_{0}(i)\right)\right\|_{l_{p}\left(A_{0}\right)},\left\|\left(2^{i(\theta-1)} a_{1}(i)\right)\right\|_{l_{p}\left(A_{1}\right)}\right\}
$$

the infimum being taken over all families $\left(a_{0}(i)\right) \subset A_{0}$ and $\left(a_{1}(i)\right) \subset A_{1}$ with $a_{0}(i)+a_{1}(i)=a$ for all $i \in \mathbb{Z}$. Then $\Delta(\vec{A}) \subset A_{\theta, p} \subset \Sigma(\vec{A})$ with continuous embeddings. The Banach space $A_{\theta, p}$ with norm $\|\cdot\|_{A_{\theta, p}}$ is called a real interpolation space with respect to $\vec{A}=\left(A_{0}, A_{1}\right)$. If $a \in A_{\theta, p}$, then

$$
\|a\|_{A_{\theta, p}} \leq 2^{\theta(1-\theta)}\left\|\left(2^{i \theta} a_{0}(i)\right)\right\|_{l_{p}\left(A_{0}\right)}^{1-\theta}\left\|\left(2^{i(\theta-1)} a_{1}(i)\right)\right\|_{l_{p}\left(A_{1}\right)}^{\theta}
$$

for all families $\left(a_{0}(i)\right) \subset A_{0}$ and $\left(a_{1}(i)\right) \subset A_{1}$ with $a_{0}(i)+a_{1}(i)=a$ for all $i \in \mathbb{Z}$ (see [11, p. 18).

Let $A_{\theta, p}$ and $B_{\theta, p}$ be two interpolation spaces with respect to the interpolation pairs $\vec{A}=\left(A_{0}, A_{1}\right)$ and $\vec{B}=\left(B_{0}, B_{1}\right)$, and let $T: \Sigma(\vec{A}) \rightarrow \Sigma(\vec{B})$ be a linear operator. We write $T: \vec{A} \rightarrow \vec{B}$, if for $j=0,1$, the restriction $T \mid A_{j}$ is a bounded operator into $B_{j}$. For every $T: \vec{A} \rightarrow \vec{B}$,

$$
\left\|T: A_{\theta, p} \rightarrow B_{\theta, p}\right\| \leq 2^{\theta(1-\theta)}\left\|T: A_{0} \rightarrow B_{0}\right\|^{1-\theta}\left\|T: A_{1} \rightarrow B_{1}\right\|^{\theta} .
$$

In the main result of this paper, we show that this classical inequality concerning boundedness has its counterpart for the ABS property.

Theorem 4.1. Let $A_{\theta, p}$ and $B_{\theta, p}$ with $0<\theta<1$ and $1<p<\infty$ be real interpolation spaces with respect to interpolation pairs $\vec{A}=\left(A_{0}, A_{1}\right)$ and $\vec{B}=\left(B_{0}, B_{1}\right)$. Then for every $T: \vec{A} \rightarrow \vec{B}$,

$$
\Phi_{A B S}\left(T: A_{\theta, p} \rightarrow B_{\theta, p}\right) \leq 2^{\theta(1-\theta)} \Phi_{A B S}^{1-\theta}\left(T: A_{0} \rightarrow B_{0}\right) \Phi_{A B S}^{\theta}\left(T: A_{1} \rightarrow B_{1}\right) .
$$

Proof. Fix $\varepsilon>0$. Let $\left(a_{n}\right)$ be a sequence in $\mathrm{B}\left(A_{\theta, p}\right)$. For each $a_{n}$ there exist $x_{j n}=\left(2^{i(\theta-j)} a_{j n}(i)\right)_{i \in \mathbb{Z}} \in \mathrm{B}\left(l_{p}\left(A_{j}\right)\right), j=0,1$, such that $a_{0 n}(i)+a_{1 n}(i)=a_{n}$ for all $i \in \mathbb{Z}$. Set $y_{j n}=\left(2^{i(\theta-j)} T a_{j n}(i)\right)_{i \in \mathbb{Z}}$ for $j=0,1$ and every $n \in \mathbb{N}$. As in the proof of subadditivity of $\Phi_{A B S}$, by Proposition 2.3. passing to a sequence of svsm built on a common sequence of svsm for $\left(a_{n}\right)$, we may assume that for all finite subsets $A \subset \mathbb{N}$ and all sequences of signs $\left(\epsilon_{n}\right)$,

$$
\left\||A|^{-1} \sum_{n \in A} \epsilon_{n} y_{j n}\right\|_{l_{p}\left(B_{j}\right)} \leq \phi_{v s m}\left(y_{j n}\right)+\varepsilon, \quad j=0,1 .
$$

Let $\widetilde{T}_{j}: l_{p}\left(A_{j}\right) \rightarrow l_{p}\left(B_{j}\right), j=0,1$, be defined as the operator $\widetilde{T}$ in Theorem 3.2 . Then $y_{j n}=\widetilde{T} x_{j n}$. It follows that

$$
\begin{aligned}
\phi_{v s m}\left(T a_{n}\right) & \leq\left\||A|^{-1} \sum_{n \in A} \epsilon_{n} T a_{n}\right\|_{B_{\theta, p}} \\
& \leq 2^{\theta(1-\theta)}\left\||A|^{-1} \sum_{n \in A} \epsilon_{n} y_{0 n}\right\|_{l_{p}\left(B_{0}\right)}^{1-\theta}\left\||A|^{-1} \sum_{n \in A} \epsilon_{n} y_{1 n}\right\|_{l_{p}\left(B_{1}\right)}^{\theta} \\
& \leq 2^{\theta(1-\theta)}\left(\phi_{v s m}\left(y_{0 n}\right)+\varepsilon\right)^{1-\theta}\left(\phi_{v s m}\left(y_{1 n}\right)+\varepsilon\right)^{\theta} \\
& \leq 2^{\theta(1-\theta)}\left(\Phi_{A B S}\left(\widetilde{T}_{0}\right)+\varepsilon\right)^{1-\theta}\left(\Phi_{A B S}\left(\widetilde{T}_{1}\right)+\varepsilon\right)^{\theta} .
\end{aligned}
$$

Since $l_{p}(X)$ with families indexed by integers is isometrically isomorphic to $l_{p}(X)$ with sequences indexed by $\mathbb{N}$, and $\phi_{v s m}$ is invariant under linear isometries, by 
Theorem 3.2, $\Phi_{A B S}\left(\widetilde{T}_{j}\right)=\Phi_{A B S}\left(T: A_{j} \rightarrow B_{j}\right), j=0,1$. By an arbitrary choice of $\varepsilon$ and $\left(a_{n}\right)$, we obtain the conclusion.

As a corollary we obtain the following generalization of Proposition IV.1 of [2] without restrictions on interpolation pairs.

Corollary 4.2. If $T: A_{0} \rightarrow B_{0}$ or $T: A_{1} \rightarrow B_{1}$ has the $A B S$ property, then so has $T: A_{\theta, p} \rightarrow B_{\theta, p}$ for all $0<\theta<1$ and $1<p<\infty$. In particular, if $A_{0}$ or $A_{1}$ has the $A B S$ property, then so has $A_{\theta, p}$.

\section{REFERENCES}

[1] K. Astala and H.-O. Tylli, Seminorms related to weak compactness and to Tauberian operators, Math. Proc. Cambridge Philos. Soc., 107 (1990), 367-375. MR.1027789 (91b:47016)

[2] B. Beauzamy, Banach-Saks properties and spreading models, Math. Scand., 44 (1979), 357384. MR555227 (81a:46018)

[3] B. Beauzamy, Espaces d'interpolation réels: Topologie et géométrie, Lecture Notes in Mathematics, 666, Springer, Berlin, 1978. MR513228 (80k:46080)

[4] B. Beauzamy, Propriété de Banach-Saks, Studia Math., 66 (1980), 227-235. MR579729 (81i:46020)

[5] A. Brunel and L. Sucheston, On B-convex Banach spaces, Math. Systems Theory, 7 (1974), 294-299. MR0438085 (55:11004)

[6] F. Cobos, A. Manzano and A. Martínez, Interpolation theory and measures related to operator ideals, Quart. J. Math. Oxford Ser. (2), 50 (1999), 401-416. MR1726783 (2000k:46104)

[7] F. Cobos and A. Martínez, Extreme estimates for interpolated operators by the real method, J. London Math. Soc. (2), 60 (1999), 860-870. MR.1753819 (2001e:46128)

[8] P. Erdös and M. Magidor, A note on regular methods of summability and the Banach-Saks property, Proc. Amer. Math. Soc., 59 (1976), 232-234. MR0430596 (55:3601)

[9] S. Heinrich, Closed operator ideals and interpolation, J. Funct. Anal., 35 (1980), 397-411. MR.563562 (81f:47045)

[10] A. Kryczka, S. Prus and M. Szczepanik, Measure of weak noncompactness and real interpolation of operators, Bull. Austral. Math. Soc., 62 (2000), 389-401. MR.1799942(2001i:46116)

[11] J.-L. Lions and J. Peetre, Sur une classe d'espaces d'interpolation, Inst. Hautes Études Sci. Publ. Math., 19 (1964), 5-68. MR0165343 (29:2627)

[12] J.R. Partington, On the Banach-Saks property, Math. Proc. Cambridge Philos. Soc., 82 (1977), 369-374. MR0448036 (56:6346)

[13] H.P. Rosenthal, Weakly independent sequences and the Banach-Saks property, in Durham symposium on the relations between infinite-dimensional and finite-dimensional convexity, Bull. London Math. Soc., 8 (1976), 1-33.

[14] H.-O. Tylli, The essential norm of an operator is not self-dual, Israel J. Math., 91 (1995), 93-110. MR1348307 (96f:47017)

Institute of Mathematics, M. Curie-SkŁodowska University, 20-031 Lublin, Poland

E-mail address: andrzej.kryczka@umcs.lublin.pl 